\title{
Diseño y validación de dos escalas de comunicación sexual con la pareja en hombres que tienen sexo con hombres
}
Design and validation of two scales of sexual communication with the partner in men who have sex with men

\section{Desenho e validação de duas escalas de comunicação sexual com o parceiro em homens que fazem sexo com homens}

\author{
Ricardo Sánchez Medina ${ }^{1}$ \\ David Javier Enríquez Negrete ${ }^{2}$ \\ Consuelo Rubi Rosales Piña ${ }^{3}$ \\ Pablo Uriel Pérez Martínez ${ }^{4}$
}

Recibido: 29 de enero de 2021

Aprobado: 6 de octubre de 2021

Publicado: 3 de noviembre de 2021

Cómo citar este artículo:

Sánchez Medina, R., Enríquez Negrete, D.J., Rosales Piña, C.R. y Pérez Martínez, P.U. (2021). Diseño y validación de dos escalas de comunicación sexual con la pareja en hombres que tienen sexo con hombres.

Pensando Psicología, 17(2), 1-31.

doi: https://doi.org/10.16925/2382-3984.2021.02.01

Artículo de investigación. https://doi.org/10.16925/2382-3984.2021.02.01

1 Universidad Nacional Autónoma de México. Facultad de Estudios Superiores Iztacala. UNAM, FES Iztacala, Torre Académica, segundo piso, cubículo 20. Av. De los Barrios $N^{\circ} 1$, Los Reyes Iztacala, Tlalnepantla, Edo. de México, México, 54090

Correo electrónico: ricardo.sanchez@iztacala.unam.mx ORCID: https://orcid.org/0000-0003-4268-3025

2 Universidad Nacional Autónoma de México. Facultad de Estudios Superiores Iztacala, Edo. de México, México.

Correo electrónico: david.enriquez@iztacala.unam.mx ORCID: https://orcid.org/0000-0002-6376-4898

3 Universidad Nacional Autónoma de México. Facultad de Estudios Superiores Iztacala, Edo. de México, México.

Correo electrónico: rubi.rosales@iztacala.unam.mx ORCID: https://orcid.org/0000-0002-0605-1859

4 Universidad Nacional Autónoma de México. Facultad de Estudios Superiores Iztacala, Edo. de México, México.

Correo electrónico: paur_permar@comunidad.unam.mx ORCID: https://orcid.org/0000-0003-4513-1648 
2 Diseño y validación de dos escalas de comunicación sexual con la pareja en hombres que tienen sexo con hombres

\section{Resumen}

Objetivo: diseñar y validar dos escalas de comunicación sexual con la pareja en hombres que tienen sexo con hombres que, en conjunto, evalúan cinco componentes de la comunicación: contenido, extensión, estilo, tiempo y contexto.

Metodología: se dividió en dos fases, en la primera se elaboran los reactivos que conforman cada escala, y en la segunda se obtuvieron sus propiedades psicométricas. En la primera fase participaron 200 hombres y, en la segunda, 1190 hombres, en ambos casos con una vida sexual activa, solteros y que reportaron tener sexo con hombres, la mitad de ellos con diagnóstico de VIH y cuya vía de infección fue a través de contacto sexual. Se aplicó la estrategia de redes semánticas naturales modificadas para obtener las principales definidoras de los estímulos: temas sexuales y comunicación sexual con la pareja. Con base en los resultados, se diseñaron los reactivos para conformar dos escalas. En ambas fases la aplicación se realizó en una clínica especializada en atención de personas con VIH.

Resultados: los resultados de la validación muestra evidencias de validez de constructo (AFE y AFC) y validez concurrente, así como buenos índices de consistencia interna $(a, \omega)$ en cada escala mostrando propiedades psicométricas adecuadas.

Conclusiones: es necesario evaluar la comunicación considerando todos sus componentes para lo cual se requiere de instrumentos culturalmente válidos como las escalas que se presentan en esta investigación.

Palabras clave: comunicación, estudio de validación, salud sexual, hombres.

\section{Abstract}

Aim: Design and validate two scales of sexual communication with the partner in men who have sex with men, which together evaluate five components of communication, content, extension, style, time and context.

Methodology: It was divided into two phases, the first to develop the items that make up each scale, and the second to obtain their psychometric properties. 200 men participated in the first phase and 1,190 men participated in the second phase, in both cases with an active sexual life, single and who reported having sex with men, half of them diagnosed with HIV and whose route of infection was through sexual contact. The strategy of modified natural semantic networks was applied to obtain the main ones defined for the stimuli: sexual themes and sexual communication with the partner, based on the results, the items were designed to form two scales. In both phases, the application was carried out in a clinic specialized in the care of people with HIV.

Results: The results of the validation show evidence of construct validity (EFA and CFA) and concurrent validity, as well as good internal consistency indexes $(a, \omega)$ in each scale showing adequate psychometric properties.

Conclusions: It is necessary to evaluate communication considering all its components, for which culturally valid instruments such as the scales presented in this research are required.

Keywords: communication, validation study, sexual health, men.

\section{Resumo}

Objetivo: desenhar e validar duas escalas de comunicação sexual com a parceira em homens que fazem sexo com homens que, juntas, avaliem cinco componentes da comunicação: conteúdo, extensão, estilo, tempo e contexto.

Metodologia: foi dividida em duas fases, na primeira são elaborados os reagentes que compõem cada escala e, na segunda, obtidas suas propriedades psicométricas. Na primeira fase participaram 200 homens e, na segunda, 1.190 homens, em ambos os casos com vida sexual ativa, solteiros e que relataram ter relações sexuais com homens, metade deles com diagnóstico de HIV e cuja via de infecção foi pela via sexual contato. 
Ricardo Sánchez Medina, David Javier Enríquez Negrete, Consuelo Rubi Rosales Piña, 3 Pablo Uriel Pérez Martínez

A estratégia de redes semânticas naturais modificadas foi aplicada para obter os principais fatores definidores dos estímulos: temas sexuais e comunicação sexual com o parceiro. Com base nos resultados, os itens foram elaborados em conformidade com duas escalas. Em ambas as fases, a aplicação foi realizada em ambulatório especializado no atendimento a pessoas com HIV.

Resultados: os resultados da validação mostram evidências de validade de construto (afe e afc) e validade concorrente, bem como bons índices de consistência interna $(a, \omega)$ em cada escala, apresentando propriedades psicométricas adequadas.

Conclusões: é necessário avaliar a comunicação considerando todos os seus componentes, para os quais são necessários instrumentos culturalmente válidos, como as escalas apresentadas nesta pesquisa.

Palavras-chave: comunicação, estudo de validação, saúde sexual, homens.

\section{Introducción}

En la actualidad algunos de los problemas de salud pública más importantes a nivel mundial son los que se refieren al tratamiento y prevención de las Infecciones de Transmisión Sexual (ITS), que son el conjunto de diversas infecciones o patologías que tienen una etiología diversa, pero cuya transmisión se da por contacto sexual (vaginal, anal u oral). Entre éstas se encuentran, la gonorrea, la sífilis, la hepatitis B, el herpes, el Virus del Papiloma Humano (VPH) y el Virus de Inmunodeficiencia Humana (VIH) (López, 2019; Otero-Guevara et al., 2017; Soto, 2015; Vidal y Hernández, 2017).

Para entender mejor el problema que representan las ITS a nivel mundial, basta con ver los datos reportados por la Organización Mundial de la Salud (OMS, 2019), que indican que cada día más de un millón de personas contraen una ITS; es decir, más de 365 millones de personas contraen una infección durante el transcurso de un año. Si bien, estos datos por sí solos ya resultan alarmantes, en el caso particular de personas infectadas por VIH se puede encontrar que, de acuerdo con las últimas estadísticas, 38 millones de personas han sido diagnosticadas con esta infección, siendo la principal vía de transmisión la sexual; de este número, solo 25.4 millones de personas tienen acceso a medicamentos para controlar la evolución del VIH (Programa Conjunto de las Naciones Unidas sobre el VIH/SIDA [ONUSIDA], 2020).

Estos datos son un indicio de actividades sexuales de riesgo, principalmente, el uso inconsistente del condón, el inicio de la actividad sexual de forma prematura, tener relaciones sexuales bajo la influencia de alcohol o drogas (Ali et al., 2019, Mengual et al., 2016). Además, este tipo de conductas suelen estar asociadas con una comunicación deficiente o poco asertiva, principalmente entre la pareja (Aholou et al., 2017; Gause et al, 2018; Li y Samp, 2019); dejando en evidencia la importancia de la comunicación sexual y su relación con conductas sexuales preventivas, como lo es el uso del condón en las relaciones sexuales; sin embargo, una de las dificultades encontradas 
corresponden a la forma de evaluarla, que hace difícil poder hacer generalizaciones de los resultados.

Al respecto Dixson (1995), Muhwezi et al. (2015) y Rogers (2017) hacen hincapié en que la comunicación debe ser considerada como una variable multidimensional con elementos como: el contenido, la extensión, el estilo, el tiempo y el contexto, permitiendo realizar una evaluación global de la comunicación. Esta forma de evaluación ha sido implementada por Cox et al. (2008) y Parker et al. (2007), definiendo los elementos antes mencionados de la siguiente forma: a) contenido, se refiere a los temas que se discuten; b) extensión, frecuencia con la que da la comunicación; c) estilo, la forma en cómo se da la comunicación; d) tiempo, temporalidad de la comunicación, es decir, en qué momento se da; y por último, d) contexto, que se refiere al tipo de situación en la cual la comunicación ocurre y las creencias que tiene de la misma. Cabe señalar que el contexto en el proceso de comunicación, tiene dos componentes el físico y el simbólico, el primero puede pensarse como el lugar bajo el cual ocurre la comunicación, o sobre las características de las personas, como ser hombre o mujer, o la orientación sexual (Ayer et al., 2021), y el segundo relacionado con el significado que le atribuyen las personas; de acuerdo con Dixson (1995), el contexto puede ser diverso o similar entre las personas, pero cómo lo perciben es lo que realmente impacta en la comunicación, es decir, el contexto influirá en cómo se estable, cómo se interpreta, además de las consecuencias que se deriven en la relación; en el caso particular de la salud sexual, permitirá conocer si ese contexto lo perciben de manera favorable o desfavorable, de qué manera se relaciona con otros componentes de la comunicación, y por último, sobre el impacto que tiene sobre el uso del condón en relaciones sexuales.

Al hacer una revisión de la literatura sobre cómo se ha evaluado la comunicación sexual, generalmente, se toma en cuenta uno de los componentes, sobre todo el contenido (Dimbuene, 2015; Fernández, et al., 2017; González et al., 2017; LópezOlmos, 2018; Uribe et al., 2016); el cual suele estar centrado en conductas sexuales, satisfacción sexual y su influencia en la díada de pareja. Esto puede verse en la Escala de Comunicación Diádica Sexual (DSC), el Cuestionario de Patrones de Comunicación Sexual S-CPQ o la Escala de Comunicación Autopercibida en la Relación de Pareja (CARP) (Anderson et al., 2016; I glesias-García et al., 2019; Jones et al., 2017; Leonhardt et al., 2018; Martínez-Huertas y Jastrzebska, 2019; Pazmany et al., 2015; Rancourt et al., 2017; Rancourt et al., 2016).

Otros instrumentos se focalizan en la importancia de la comunicación sexual, pero en la relación padres e hijos, mostrando que cuando los padres hablan con sus hijos acerca de sexualidad (contenido de la comunicación), es más probable que estos 
tengan un comportamiento sexual preventivo, como usar condón en relaciones sexuales o posponer sus encuentros sexuales; algunos instrumentos utilizados son la Escala de Comunicación de Riesgos Sexuales entre Padres y Adolescente (PTSRC-III por sus siglas en inglés) y la Escala de Comunicación Padres-Adolescentes (PACs, por sus siglas en inglés) (Araujo-Robles et al., 2018; Carrascosa et al., 2015; Esparza et al., 2017; Gabbidon et al., 2017; Harris, 2016; Tuason et al., 2017; Widyatuti et al., 2018).

En el caso de la comunicación sexual con la pareja, generalmente se evalúa el estilo que utilizan; por ejemplo, la conducta verbal y no verbal en la comunicación con la pareja. En estos trabajos se señala que no basta con evaluar lo que dicen las personas, sino cómo el lenguaje no verbal tiene un impacto sobre la conducta sexual (Blunt-Vinti et al., 2019; Santos-Iglesias y Byers, 2020). Otros estilos de comunicación que se han evaluado están centrados en los estilos de negociación del uso del condón, principalmente el asertivo, la forma de evaluar ha sido a través de entrevistas semiestructuradas (Aholou et al., 2017) o cuestionarios autoaplicables (Megaputri et al., 2019). Hay quienes consideran la comunicación como una habilidad, y en ocasiones se mide a partir de indicadores de autoeficacia (Dharma et al., 2019) o a través de la evaluación de la habilidad mediante medidas de autoreporte (Peasant et al., 2017) o de observación directa (Sánchez et al., 2018).

Con base en lo anterior, se observa que la forma en que se evalúa la comunicación y los componentes que se consideran es diversa, si bien dichas investigaciones contribuyen en la explicación de la relación de la comunicación sexual con la conducta sexual, es necesario contemplar el constructo en todas sus dimensiones para tener indicadores más precisos en su evaluación (Martínez et al., 2006).

Al respecto, investigaciones que consideran en su formulación teórica la multidimensionalidad de la comunicación son los trabajos de Álvarez y Villaruel (2015) quienes evalúan a jóvenes sobre diferentes tipos de comunicación sexual; en cambio Quinn-Nilas et al. (2015) evalúan el contenido y los estilos de la comunicación, pero a través de la autoeficacia, otros autores mencionan la importancia de la comunicación entre padres e hijos y presentan las propiedades psicométricas de instrumentos de medición para evaluar este constructo considerando su multidimensionalidad, por ejemplo, Rogers et al. (2020) obtuvieron los siguientes factores: frecuencia de la comunicación, calidad de la comunicación sexual y mensajes sobre sexo (contenido), Cox et al. (2008) encontraron los siguientes factores, contenido, contexto, momento y estilo. Estos autores resaltan la importancia de la multidimensionalidad de la comunicación entre padres e hijos y su relación con la conducta sexual para promover la salud sexual. 
En el caso particular de adultos, se encontró una revisión sistemática sobre cómo es la comunicación en personas que se encuentran en etapas avanzadas de la enfermedad y sus cuidadores; Parker et al. (2007) encontraron que la comunicación tiene un papel importante en la calidad de vida de los enfermos, principalmente, sobre el contenido, el estilo utilizado y el momento en el que se da la comunicación; estos resultados muestran la importancia que tiene la comunicación, para que las personas tengan conductas de prevención; sin embargo, es necesario mostrar evidencia sobre la comunicación sexual con la pareja, y al mismo tiempo poner atención a ciertos grupos que, por el contexto en el que se encuentran, son más susceptibles, como las personas con VIH (ONUSIDA, 2020).

En este contexto, se busca que quienes ya están infectados puedan mejorar su calidad de vida a través de una buena adherencia al tratamiento (Altice et al., 2019), pero que al mismo tiempo que tengan una conducta sexual preventiva para evitar reinfecciones o nuevas infecciones por VIH; por lo que es necesario diseñar intervenciones para este sector de la población que es sexualmente activa para promover el uso del condón en relaciones sexuales (Ali et al., 2019), en este mismo orden de ideas ONUSIDA (2020) afirma que son los hombres quienes más se infectan, y entre ellos es mayor la proporción de hombres que tienen sexo con hombres (HSH). Con base en lo anterior, se requiere contar con instrumentos culturalmente válidos sobre comunicación sexual con la pareja, atendiendo a las características del contexto en el que se encuentran, en especial con HSH y con diagnóstico de $\mathrm{VIH}$, de tal forma que un segundo momento se pueda hacer evaluación diagnóstica e intervenciones eficaces (Francis et al., 2018; Francis et al., 2019).

En el caso particular de HSH en que es más frecuente la comunicación sobre $\mathrm{VIH}$, es entre quienes se identifican como heterosexuales, que entre los que se consideran bisexuales o gais (Ayer et al., 2021). Dentro de los HSH también hay diferencias en términos del diagnóstico de VIH, por ejemplo, los estereotipos y el estigma del VIH es mayor en VIH positivos y esto influye en la poca comunicación con la pareja sexual (Haas et al., 2020), de igual forma, como mencionan Grimm y Schwartz (2021), la comunicación es fundamental para que los HSH tengan conductas preventivas, por ejemplo, se ha observado que HSH que se comunican más con su pareja tienen menores conductas de riesgo sexual (Afriyanti et al., 2020; Crosby et al., 2016; Gause, et al., 2018; Lo et al., 2011).

Para finalizar, los instrumentos revisados que evalúan comunicación sexual en la pareja permiten derivar las siguientes conclusiones; en primer lugar, el componente de la comunicación más utilizado en la evaluación es el contenido; en segundo lugar, la muestra evaluada comprende a adolescentes o jóvenes que se encuentran cursando 
algún grado escolar o sin experiencia sexual; en tercer lugar, es necesario evaluar la multidimensionalidad de la comunicación que permita ampliar el foco de análisis para comprender cómo es el proceso en la pareja. Tomando en cuenta lo anterior, el propósito de la presente investigación fue diseñar y validar dos escalas de comunicación sexual con la pareja en hombres que tienen sexo con hombres, con base en la propuesta de Dixson (1995).

\section{Método}

\section{Fase 1. Construcción del instrumento}

\section{Participantes}

Se trabajó con 200 hombres que reportaron una vida sexual activa, tener relaciones sexuales con hombres y ser solteros. El 50\% de ellos tenían un diagnóstico de VIH, en que la vía de transmisión fue el contacto sexual. A través de un muestreo por conveniencia (Kerlinger y Lee, 2002), se invitó a los usuarios de una clínica especializada en atención de $\mathrm{VIH}$ a responder un cuestionario. Los hombres con diagnóstico de VIH tenían una edad promedio de 27,31 años (DE=8,86), el 3,16\% contaba con estudios de primaria, el 10,53\% de secundaria, el 34,74\% de carrera técnica, el 44,21\% de universidad y el 7,.37\% de posgrado. El promedio de tiempo de diagnóstico de VIH es de 3,57 años (DE=5,19). Los hombres sin diagnóstico de $\mathrm{VIH}$ reportaron una edad promedio de 23,15 años (DE=6,31), el 19\% tenía estudios de secundaria, el 16\% de carrera técnica, el $57 \%$ de universidad y el $8 \%$ de posgrado.

\section{Instrumentos}

Se utilizó la estrategia de Redes Semánticas Naturales Modificadas (RSNM) de ReyesLagunes (1993) que consiste en identificar significados o conductas que las personas les atribuyen a ciertos estímulos. En la presente investigación, estos estímulos fueron temas sexuales y comunicación sexual con la pareja, además de un estímulo distractor; los estímulos se presentaron en orden aleatorio para evitar un efecto de acarreo. Cada estímulo se presentó en la parte superior de una hoja y se indicó a los participantes que debían describir cada estímulo con al menos cinco palabras y que al terminar las enumeraran en función de la importancia que ellos le atribuían, asignando el número uno, a aquella que consideran más relevante y así sucesivamente. El 
uso de esta estrategia ha mostrado evidencia empírica en la construcción de instrumentos de medición culturalmente relevantes, en diversas poblaciones y constructos (Bouquet et al., 2019; Campos-Rivera \& Sotelo-Quiñonez, 2019; Capilla-Garrido et al., 2020; Enríquez et al., 2021; Fonseca et al., 2019; García-Torres et al., 2017; Granados, 2019; Irepan y Ortega, 2020; Reyes et al., 2017; Rosales et al., 2019).

\section{Procedimiento}

Se estableció un convenio con una clínica especializada en la atención de personas con VIH. La aplicación de instrumentos se realizó de manera individual en el área en que los hombres esperan para ser atendidos o para solicitar una prueba de detección de VIH. En ambos casos, se hizo énfasis en la participación voluntaria, así como en el anonimato y confidencialidad de la información a través de un consentimiento informado, a quienes aceptaron se les proporcionó el instrumento; en promedio tardaron 20 minutos en responderlo.

\section{Análisis de resultados}

Para el diseño de cada uno de los reactivos de las dos escalas, se siguió el procedimiento de Reyes-Lagunes (1993). Primero, se formaron dos grupos de hombres con diagnóstico de VIH y hombres sin el diagnóstico, debido a que en la literatura se ha reportado que el conocimiento del estado serológico influye en la manera en que se comunican con la pareja, así como en el comportamiento sexual (Ayer et al., 2021; Damian et al., 2019; Gursahaney et al., 2019; Hass et al., 2017; Ncube, et al., 2017). El análisis se realizó en hojas de cálculo en Excel. Cada estímulo fe analizado por separado. Primero, se obtuvo el número total de palabras definidoras o tamaño de la red (TR); segundo, se conseguió el peso semántico (PS) a partir de la suma de frecuencias de cada palabra y del orden de importancia que le atribuyen; tercero, se ordenó de mayor a menor, las primeras palabras indican cuáles son aquellas palabras con mayor frecuencia; cuarto, se obtuvo la distancia semántica cuantitativa (DSC), para lo que se asigna el porcentaje más alto a aquella definidora con mayor Ps (100\%), por regla de tres se determinan los valores restantes; posteriormente, se recodifican los valores de cero a 100 y por regla de tres se cambia el resto de los valores. Las palabras más cercanas a cero significan que tienen mayor significado y conforme se van alejando, lo va perdiendo. Para determinar si se requería un instrumento o dos en función del diagnóstico de VIH, de acuerdo con Reyes-Lagunes (1993) e Hinojosa (2008) se obtiene un análisis de consenso grupal a través de un análisis de diferencias de 
las palabras en términos de su carga afectiva (descriptivas, positivas y negativas) a través de una ji cuadrada, si existen diferencias entre los grupos se requiere un instrumento diferente por grupo debido a que el significado es diferente y por lo cual podría no ser culturalmente válido; adicionalmente se realizó un análisis de correlación de Spearman para identificar el número de palabras en las que concuerdan e identificar el peso semántico que les atribuyen a las palabras, de tal manera que se pueda tener un indicador de qué tanto coinciden los grupos. Para profundizar sobre las aplicaciones e interpretación del análisis de resultados se puede consultar a Pérez (2015) y Valdez (1998). Con base en los resultados obtenidos se diseñaron y construyeron dos escalas de comunicación, que fueron utilizadas en la fase 2 para obtener sus propiedades psicométricas.

\section{Fase 2. Validación del instrumento}

\section{Participantes}

Se trabajó con 1190 hombres que reportaron una vida sexual activa, tener relaciones sexuales con hombres y ser solteros. El 52,7\% tenía un diagnóstico de VIH cuya vía de transmisión fue sexual. A través de un muestreo por conveniencia (Kerlinger y Lee, 2002) se invitó a los usuarios de una clínica especializada en atención de VIH. Los hombres con diagnóstico de VIH tenían una edad promedio de 33,81 años ( $D E=9,32)$, $3,8 \%$ tenía estudios de primaria, $11,9 \%$ de secundaria, $34,9 \%$ de preparatoria, $44,7 \%$ de universidad y $6,5 \%$ de posgrado. El promedio de tiempo de diagnóstico de VIH es de 5,48 años ( $\mathrm{DE}=5,79$ ); el $81 \%$ con orientación homosexual, $11 \%$ bisexual y $8.1 \%$ heterosexual. Los hombres sin diagnóstico de VIH reportaron una edad promedio de 27,92 años ( $D E=7,86)$, el 0,9\% con estudios de primaria, 9,7\% con estudios de secundaria, $38,2 \%$ de preparatoria, $42,3 \%$ de universidad, $8,1 \%$ de posgrado. Su orientación sexual se distribuyó en $59,6 \%$ homosexual, $14,9 \%$ bisexual y $25,4 \%$ heterosexual.

\section{Tipo y diseño}

El tipo de estudio es instrumental debido a que está centrado en el análisis de las propiedades psicométricas de las escalas de medición (Montero y León, 2007). El tipo de diseño de investigación es transversal, ya que únicamente se recolectan datos en un solo momento (Kerlinger y Lee, 2002). 


\section{Instrumentos}

Con base en los resultados obtenidos en la fase 1, se diseñaron dos escalas para evaluar los componentes de la comunicación centrados en el contenido, estilo y contexto:

Escala sobre temas sexuales con la pareja. Conformada por 15 reactivos que evalúan la frecuencia con la que hablan sobre diversos temas sexuales con su pareja. Las opciones de respuesta están en formato tipo likert que van de nunca (1), casi nunca (2), algunas veces (3), casi siempre (4) y siempre (5).

Escala sobre comunicación sexual con la pareja. Conformada por 12 reactivos que evalúan la frecuencia con respecto a cómo se siente cada vez que habla con su pareja sobre algún tema relacionado con su vida sexual. Las opciones de respuesta están en formato tipo likert que van de nunca (1), casi nunca (2), algunas veces (3), casi siempre (4) y siempre (5).

Adicionalmente, para la validez concurrente, se evaluó:

Consistencia en el uso del condón. Dos reactivos para evaluar el número de veces que tuvieron relaciones sexuales y usaron condón en los últimos tres meses. Con base en la propuesta de DiClemente y Wingood (1995), se obtiene un índice de consistencia con valores entre cero y uno, que es resultado de dividir el número de veces de uso de condón en tres meses entre el número de relaciones sexuales, en ese mismo periodo de tiempo. Valores iguales a uno se indica que la persona es consistente en el uso del condón.

\section{Procedimiento}

Se estableció un convenio con una clínica especializada en la atención de personas con VIH, la aplicación de instrumentos se realizó de manera individual en el área en que los hombres esperan para ser atendidos o para solicitar una prueba de detección de VIH. En ambos casos, se hizo énfasis en la participación voluntaria, así como en el anonimato y confidencialidad de la información a través de un consentimiento informado. Quienes aceptaron se les proporcionó el instrumento; en promedio, tardaron 20 minutos en responder.

\section{Análisis de resultados}

Los datos fueron analizados en el paquete SPSS versión 26 y el programa Jamovi versión 2.0. Para la validez de constructo en cada una de las escalas, se realizó un análisis discriminante por reactivo para valorar la sensibilidad del reactivo en relación con toda la escala mediante una prueba $t$ de student para datos no relacionados. 
Posteriormente, el análisis se llevó a cabo por ítem, un análisis de la asimetría, curtosis y correlación corregida del ítem con la puntuación total. Únicamente los reactivos que fueron significativos en el análisis discriminante y que tuvieron valores entre -2 y 2 en la asimetría (Lloret-Segura et al., 2014) fueron considerados para el Análisis Factorial Exploratorio (AFE) a través del método de rotación Oblimin. Previo a esto, se realizó un análisis paralelo para determinar el número de factores a extraer. Con base en estos resultados de los factores extraídos con valor propio mayor de 1 y con cargas factoriales mayores de 0,40 se incluyeron en el Análisis Factorial Confirmatorio (AFC), tomando en consideración los siguientes parámetros para estimar el modelo: CFI con valores mayores de 0,90 (ajuste adecuado) o 0,95 (buen ajuste), RMSEA con valores menores de 0,08 (ajuste adecuado) o 0,05 (buen ajuste), sRMR con valores menores a 0,5 (Abad et al., 2011; Schumacker y Lomax, 2016). Con base en estos análisis, se estimó el índice de consistencia interna para cada escala, a través del coeficiente alfa y del coeficiente omega, tomando como criterio 0,70 como un valor aceptable, y valores por encima de 0,80 adecuados (Olaz, 2014; Reidl-Martínez, 2013). Adicionalmente, se realizó un análisis de correlación entre las escalas, así como con la consistencia en el uso del condón para obtener la validez concurrente mediante un análisis de correlación de Pearson. Por último, para valorar el componente de la comunicación centrado en el tiempo, es decir, sobre el momento en el que se da la comunicación, se realizó una comparación entre hombres con y sin diagnóstico de VIH, a través de una $t$ de student para datos no relacionados.

\section{Resultados}

\section{Fase 1. Construcción del instrumento}

En la figura 1 se observan las principales palabras definidoras otorgadas por los participantes al estímulo temas sexuales, en el caso de los hombres con diagnóstico de VIH se observa que los tres principales temas son ITS, sexo e información; mientras que en los hombres con diagnóstico de VIH los temas son sexo, amor y condón, si bien comparten algunas palabras el orden que le atribuyen es diferente. Al hacer un análisis de diferencias entre las palabras descriptivas, positivas y negativas, no se encontraron diferencias significativas entre los grupos, pero sí una correlación positiva entre el peso semántico que le atribuyen $\left(r_{s}=0,75, p<0,05\right)$. 
Diseño y validación de dos escalas de comunicación sexual con la pareja en hombres que tienen sexo con hombres
a) Hombres con diagnóstico de VIH
b) Hombres sin diagnóstico de VIH
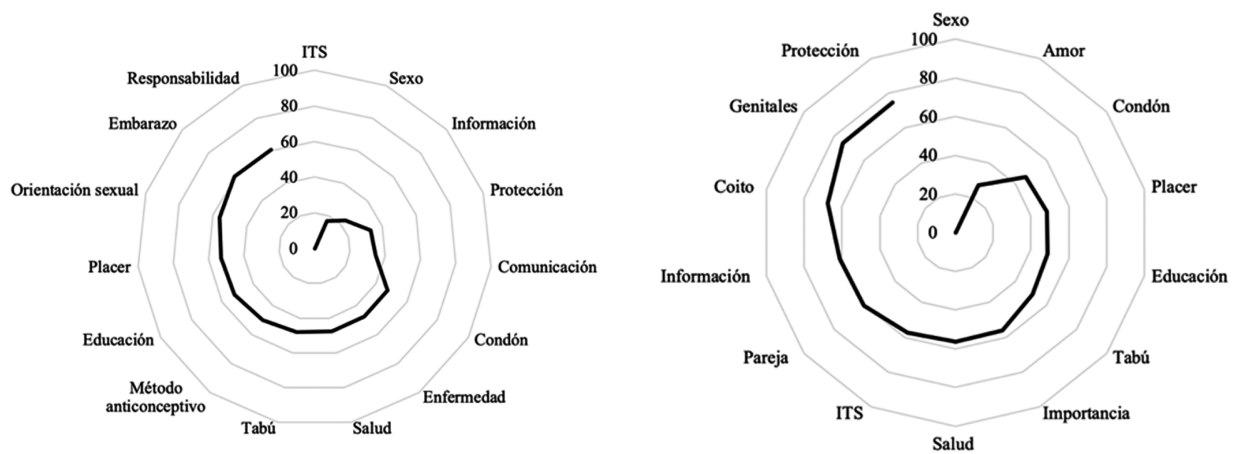

Figura 1. Principales definidoras otorgadas al estímulo Temas sexuales.

Fuente: elaboración propia.

En la figura 2 se presentan las principales palabras definidoras al estímulo comunicación sexual con la pareja, tanto los hombres con y sin VIH coinciden en las palabras confianza, amor y respeto, pero en distinto orden; también se observa que la percepción de la comunicación entre los grupos es diferente, de igual forma no se encontraron diferencias significativas con respecto a la clasificación de las palabras en descriptivas, positivas y negativas; aunque tampoco hubo una relación entre el peso que le atribuyen a la comunicación en la pareja.

a) Hombres con diagnóstico de VIH

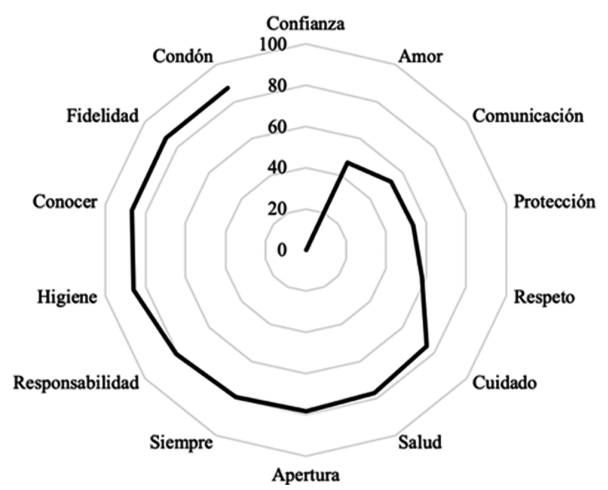

b) Hombres sin diagnóstico de VIH

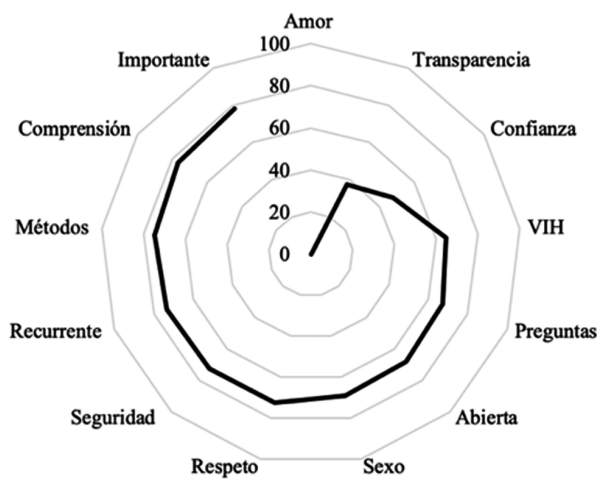

Figura 2. Principales definidoras otorgadas al estímulo Comunicación sexual con la pareja.

Fuente: elaboración propia. 
Con base en los resultados obtenidos en las redes semánticas, se decidió construir dos instrumentos de medición para ambos grupos, hombres con y sin diagnóstico de VIH centrados en los componentes de la comunicación propuestos por Dixson (1995) centrados en el contenido, estilo y contexto.

\section{Fase 2. Validación del instrumento}

\section{Escala de temas sexuales (AFE y AFC)}

En la tabla 1 se presentan los resultados obtenidos del análisis discriminante para cada uno de los reactivos de la escala de temas sexuales, así como los valores de asimetría y curtosis. Grosso modo se observa que en todos los reactivos las diferencias entre las medias del grupo con puntaje bajo y del grupo con puntaje alto, fueron estadísticamente significativas. Los valores de asimetría, curtosis y correlación corregida del ítem con la puntuación total también son adecuados.

Tabla 1. Análisis discriminante y asimetría por reactivo de la escala de temas sexuales.

\begin{tabular}{ccccccccc}
\hline & \multicolumn{2}{c}{ Grupo bajo (n=253) } & \multicolumn{2}{c}{ Grupo alto (n=249) } & \multicolumn{3}{c}{ Toda la muestra (n=1190) } \\
\hline Reactivo & Media & DE & Media & DE & $\boldsymbol{t}$ & Asimetría & Curtosis & Correlación $^{\mathbf{a}}$ \\
\hline 1 & 3,25 & 1,29 & 4,75 & 0,60 & $16,62^{*}$ & $-1,113$ & 0,552 & 0,544 \\
\hline 2 & 3,40 & 1,20 & 4,84 & 0,46 & $17,59^{*}$ & $-1,119$ & 0,819 & 0,537 \\
\hline 3 & 2,65 & 1,26 & 4,84 & 0,57 & $24,70^{*}$ & $-0,799$ & $-0,462$ & 0,588 \\
\hline 4 & 2,36 & 1,05 & 4,94 & 0,26 & $33,04^{*}$ & $-0,649$ & $-0,593$ & 0,727 \\
\hline 5 & 1,58 & 0,94 & 4,61 & 1,10 & $37,30^{*}$ & 0,106 & $-1,690$ & 0,475 \\
\hline 6 & 2,25 & 1,06 & 4,93 & 0,42 & $36,95^{*}$ & $-0,683$ & $-0,753$ & 0,672 \\
\hline 7 & 2,48 & 1,05 & 5,00 & 0,06 & $37,61^{*}$ & $-1,048$ & 0,190 & 0,761 \\
\hline 8 & 2,95 & 1,17 & 4,99 & 0,10 & $27,17^{*}$ & $-1,259$ & 0,923 & 0,713 \\
\hline 9 & 2,40 & 1,03 & 4,85 & 0,62 & $32,01^{*}$ & $-0,626$ & $-0,838$ & 0,620 \\
\hline 10 & 1,76 & 1,00 & 4,88 & 0,48 & $44,36^{*}$ & $-0,315$ & $-1,457$ & 0,554 \\
\hline 11 & 3,19 & 1,25 & 4,96 & 0,20 & $16,82^{*}$ & $-1,328$ & 1,161 & 0,655 \\
\hline 12 & 2,61 & 1,08 & 4,98 & 0,16 & $22,06^{*}$ & $-0,999$ & 0,147 & 0,744 \\
\hline 13 & 3,27 & 1,20 & 4,98 & 0,12 & $34,22^{*}$ & $-1,357$ & 1,408 & 0,664 \\
\hline 14 & 2,75 & 1,13 & 5,00 & 0,06 & $22,34^{*}$ & $-1,166$ & 0,609 & 0,763 \\
\hline 15 & 2,44 & 1,33 & 4,95 & 0,26 & $31,00^{*}$ & $-0,871$ & $-0,520$ & 0,621 \\
\hline
\end{tabular}

Nota. ${ }^{\star} p<0,001$, aCorrelación corregida del ítem con la puntuación total. Fuente: elaboración propia. 
Posteriormente, se realizó un análisis paralelo para determinar el número de factores a extraer. Dicho análisis mostró que son dos los factores que deben solicitarse para el AFE. Se aplicaron las pruebas KMO de adecuación muestral $(0,944)$ y de esfericidad de Bartlett (10248,876), resultando significativas $(p<0,001)$, por lo que los quince reactivos de la escala se sometieron al AFE. En la tabla 2 se presentan los resultados obtenidos por los dos factores, el primero de ellos con nueve reactivos, y el segundo con cinco, únicamente se eliminó el reactivo siete debido a que compartía carga factorial; al final de la tabla se presenta la varianza explicada por factor, en total ambos factores explican el $64,3 \%$ de la varianza.

Tabla 2. Validez de constructo y consistencia interna de la escala de temas sexuales.

\begin{tabular}{|c|c|c|c|}
\hline \multirow{2}{*}{ İtem } & \multirow{2}{*}{ Contenido del reactivo } & \multicolumn{2}{|c|}{ Carga factorial } \\
\hline & & 1 & 2 \\
\hline & Factor 1. Temas centrados en salud sexual & & \\
\hline 10 & Métodos anticonceptivos & 0,895 & \\
\hline 5 & Consecuencias de los embarazos & 0,860 & \\
\hline 6 & Cuidado de los genitales & 0,804 & \\
\hline 12 & Prevención al tener relaciones sexuales & 0,722 & \\
\hline 4 & Educación sexual & 0,713 & \\
\hline 14 & Responsabilidad al tener sexo & 0,679 & \\
\hline 3 & Usar condón & 0,675 & \\
\hline 15 & Orientación sexual & 0,573 & \\
\hline \multirow[t]{2}{*}{9} & Infecciones de transmisión sexual & 0,566 & \\
\hline & Factor 2. Temas centrados en relaciones sexuales & & \\
\hline 2 & Tener relaciones sexuales & & 0,877 \\
\hline 13 & Sexo & & 0,752 \\
\hline 1 & Amor en la relación de pareja & & 0,741 \\
\hline 11 & Placer sexual & & 0,603 \\
\hline \multirow[t]{3}{*}{8} & Intimidad en la pareja & & 0,598 \\
\hline & Valores eigen & 8,27 & 1,37 \\
\hline & \% varianza explicada & 40,7 & 23,6 \\
\hline
\end{tabular}

Fuente: elaboración propia.

Con base en los resultados obtenidos se realizó el AFC, en la tabla 3 se observa que el primer modelo no se tuvo un buen ajuste del modelo en RMSEA, por lo cual se procedió con la reespecificación del modelo, de acuerdo con los índices de modificación, solo bastó con correlacionar los errores de los reactivos 5 y 10, de tal forma que el AFC a través del modelo 2 se ajustó de manera adecuada. 
Ricardo Sánchez Medina, David Javier Enríquez Negrete, Consuelo Rubi Rosales Piña, 15 Pablo Uriel Pérez Martínez

Tabla 3. Índices de bondad de ajuste por modelo de la escala de temas sexuales.

\begin{tabular}{lcccccccc}
\hline & $\boldsymbol{x}^{\mathbf{2}}$ & $\mathbf{g l}$ & $\begin{array}{c}\mathbf{p} \text {-valor } \\
\left(\boldsymbol{x}^{\mathbf{2}}\right)\end{array}$ & $\mathbf{C F I}$ & RMSEA & SRMR & AIC & BIC \\
\hline Modelo 1 & 880 & 7 & $<0,001$ & 0,91 & 0,09 & 0,04 & 43147 & 43364 \\
\hline Modelo 2 & 696 & 75 & $<0,001$ & 0,93 & 0,08 & 0,04 & 4296 & 4318 \\
\hline
\end{tabular}

Nota. aReespecificación del modelo al correlacionar los errores de los ítems 5 y 10. Fuente: elaboración propia.

\section{Escala sobre comunicación sexual con la pareja (AFE y AFC)}

El mismo procedimiento se realizó con la escala de comunicación sexual con la pareja. En la tabla 4 se presenta el análisis discriminante para cada uno de los reactivos, en donde se observa que en todos hubo diferencias significativas, de igual forma se presenta el análisis de asimetría, curtosis y de correlación del ítem con la puntuación total, grosso modo se observan valores adecuados, con excepción de los valores de la curtosis en los reactivos 5 y 12 .

Tabla 4. Análisis discriminante y asimetría por reactivo de la escala sobre comunicación sexual con la pareja.

\begin{tabular}{ccccccccc}
\hline & \multicolumn{2}{c}{ Grupo bajo (n=275) } & \multicolumn{2}{c}{ Grupo alto (n=288) } & \multicolumn{3}{c}{ Toda la muestra (n=1190) } \\
\hline Reactivo & Media & DE & Media & DE & $\boldsymbol{t}$ & Asimetría & Curtosis & Correlación $^{\mathbf{a}}$ \\
\hline 1 & 2,97 & 1,13 & 4,97 & 0,16 & $29,66^{*}$ & $-1,251$ & 0,793 & 0,656 \\
\hline 2 & 2,81 & 1,04 & 4,97 & 0,17 & $34,60^{*}$ & $-1,040$ & 0,367 & 0,654 \\
\hline 3 & 2,91 & 1,04 & 4,99 & 0,10 & $33,52^{*}$ & $-1,306$ & 1,071 & 0,684 \\
\hline 4 & 3,07 & 1,04 & 5,00 & 0,05 & $31,22^{*}$ & $-1,475$ & 1,581 & 0,715 \\
\hline 5 & 3,53 & 1,14 & 5,00 & 0,00 & $21,78^{*}$ & $-1,809$ & 3,056 & 0,642 \\
\hline 6 & 3,07 & 1,01 & 4,99 & 0,10 & $31,86^{*}$ & $-1,341$ & 1,312 & 0,691 \\
\hline 7 & 2,81 & 0,99 & 5,00 & 0,00 & $37,41^{*}$ & $-1,173$ & 0,653 & 0,665 \\
\hline 8 & 2,84 & 1,14 & 4,99 & 0,08 & $31,77^{*}$ & $-1,212$ & 0,588 & 0,622 \\
\hline 9 & 2,48 & 1,12 & 4,97 & 0,17 & $36,92^{*}$ & $-0,747$ & $-0,621$ & 0,575 \\
\hline 10 & 3,43 & 1,18 & 4,98 & 0,13 & $21,98^{*}$ & $-1,332$ & 1,243 & 0,546 \\
\hline 11 & 3,08 & 1,16 & 4,99 & 0,11 & $27,76^{*}$ & $-1,389$ & 1,317 & 0,556 \\
\hline 12 & 3,44 & 1,28 & 5,00 & 0,00 & $20,71^{*}$ & $-1,926$ & 3,149 & 0,538 \\
\hline
\end{tabular}

Nota. ${ }^{\star} p<0,001$, aCorrelación corregida del ítem con la puntuación total. Fuente: elaboración propia.

El análisis paralelo mostró que dos factores se desprenden de estos 12 reactivos, con base en el análisis de adecuación muestral KMO $(0,952)$ y de la prueba de esfericidad de Bartlett $(10007,920)$ que resultaron significativas $(p<0,001)$, se procedió 
con el AFE. En la tabla 5 se observan los resultados obtenidos de estos dos factores, la escala total tuvo una varianza explicada de 65,7\%; únicamente se eliminó el reactivo 2 y 10 , por no tener carga factorial mayor a 0,40 .

Tabla 5. Validez de constructo y consistencia interna sobre comunicación sexual con la pareja.

\begin{tabular}{|c|c|c|c|}
\hline \multirow{2}{*}{ Ítem } & \multirow{2}{*}{ Contenido del reactivo } & \multicolumn{2}{|c|}{ Carga factorial } \\
\hline & & 1 & 2 \\
\hline & Factor 1. Estilo de comunicación asertiva & & \\
\hline 7 & Me siento comprendido al hablar de temas sexuales & 0,901 & \\
\hline 3 & Puedo expresar libremente mis ideas & 0,839 & \\
\hline 8 & Es fácil tener una conversación sincera & 0,794 & \\
\hline 4 & Puedo conversar sobre temas de sexualidad & 0,754 & \\
\hline \multirow[t]{2}{*}{1} & Le tengo confianza para hablar sobre mi conducta sexual & 0,506 & \\
\hline & Factor 2. Contexto favorable de la comunicación sexual & & \\
\hline 12 & Le tengo confianza para hablar sobre mi orientación sexual & & 0,842 \\
\hline 6 & Respeta mis puntos de vista sobre la sexualidad & & 0,817 \\
\hline 9 & Me ha enseñado temas de sexualidad & & 0,673 \\
\hline 11 & Me siento cómodo al hablar de temas sexuales & & 0,565 \\
\hline \multirow[t]{3}{*}{5} & Es importante hablar sobre temas sexuales & & 0,468 \\
\hline & Valores eigen & 6,87 & 0,22 \\
\hline & \% varianza explicada & 50,7 & 14,9 \\
\hline
\end{tabular}

Fuente: elaboración propia.

Con base en los 10 reactivos se realizó el AFC, en la tabla 6 se observan los resultados del primer modelo, en la que se observa que el resultado obtenido en RMSEA no permite mostrar el ajuste del modelo, por lo que se procedió a la reespecificación del modelo, en este segundo análisis se correlacionaron los errores de los reactivos 11 y 12, pero nuevamente no fue suficiente, por lo que el tercer modelo además de la relación de estos errores también se hizo entre los reactivos 7 y 8 , con base en estos resultados, el modelo 3 mostró un buen ajuste del modelo. 
Ricardo Sánchez Medina, David Javier Enríquez Negrete, Consuelo Rubi Rosales Piña,

Tabla 6. Índices de bondad de ajuste por modelo de la escala de comunicación sexual con la pareja.

\begin{tabular}{lcccccccc}
\hline & $\boldsymbol{x}^{\mathbf{2}}$ & $\mathbf{g l}$ & $\begin{array}{c}\mathbf{p} \text {-valor } \\
\left(\boldsymbol{x}^{\mathbf{2}}\right.\end{array}$ & CFI & RMSEA & SRMR & AIC & BIC \\
\hline Modelo 1 & 427 & 34 & $<0,001$ & 0.95 & 0,10 & 0,02 & 25473 & 25629 \\
\hline${\text { Modelo } 2^{\mathrm{a}}}^{2}$ & 363 & 33 & $<0,001$ & 0.96 & 0,09 & 0,02 & 25412 & 25573 \\
\hline Modelo 3 $^{\mathrm{b}}$ & 317 & 32 & $<0,001$ & 0.96 & 0,08 & 0,02 & 25367 & 25533 \\
\hline
\end{tabular}

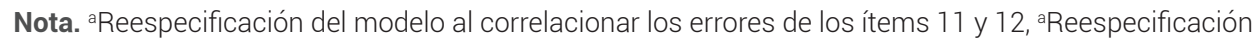
del modelo al correlacionar los errores de los ítems 7 y 8 . Fuente: elaboración propia.

\section{Validez concurrente de las escalas}

Para obtener la validez concurrente en la tabla 7 se presenta el análisis de relación entre las dos escalas validadas, así como la relación de cada escala con la consistencia en el uso del condón, se observa que en todos los casos la correlación es positiva y significativa.

Tabla 7. Validez concurrente entre los factores de las escalas de comunicación y consistencia en el uso del condón.

\begin{tabular}{|c|c|c|c|c|c|}
\hline & 1 & 2 & 3 & 4 & 5 \\
\hline 1. Consistencia en el uso del condón & - & & & & \\
\hline 2. Temas centrados en salud sexual & $0,298^{*}$ & - & & & \\
\hline 3. Temas centrados en relaciones sexuales & $0,106^{*}$ & $0,695^{*}$ & - & & \\
\hline 4. Estilo de comunicación asertiva & $0,147^{\star}$ & $0,485^{*}$ & $0,535^{*}$ & - & \\
\hline 5. Contexto favorable de la comunicación sexual & $0,113^{*}$ & $0,485^{*}$ & $0,535^{*}$ & $0,872^{*}$ & - \\
\hline
\end{tabular}

Nota. * $p<0,01$. Fuente: elaboración propia.

\section{Análisis de consistencia interna}

Para el análisis de consistencia interna de las dos escalas, se utilizó el coeficiente alfa de Cronbach y el coeficiente omega de McDonald, en la tabla 8 se observa que se obtuvieron valores superiores a 0,80 con ambos índices de consistencia, los valores obtenidos indican que se obtuvo un buen nivel de consistencia interna en cada factor de las dos escalas. 
Tabla 8. Índice de consistencia interna de las escalas.

\begin{tabular}{lll}
\hline & $\boldsymbol{\alpha}$ & $\boldsymbol{\omega}$ \\
\hline Escala de temas sexuales & & 0,91 \\
\hline Temas centrados en salud sexual & 0,90 & 0,86 \\
\hline Temas centrados en relaciones sexuales & 0,86 & \\
\hline Comunicación sexual con la pareja & & 0,91 \\
\hline Estilo de comunicación asertiva & 0,91 & 0,86 \\
\hline Contexto favorable de la comunicación sexual & 0,84 & \\
\hline
\end{tabular}

Nota. $a=$ Coeficiente alfa de Cronbach, $\omega=$ Coeficiente omega de McDonald. Fuente: elaboración propia

Por último, para el componente de la comunicación referida al tiempo, en la tabla 9 se presenta un análisis de diferencias entre los hombres con y sin diagnóstico de VIH, se observa que para ambas escalas hubo diferencias significativas, siendo ligeramente más alta la frecuencia con la que hablan con su pareja, así como la forma en cómo perciben dicha comunicación en los hombres sin diagnóstico de VIH.

Tabla 9. Comparación por grupo de acuerdo con el momento en que se da la comunicación con la pareja.

\begin{tabular}{|c|c|c|c|c|c|}
\hline & \multicolumn{2}{|c|}{$\begin{array}{l}\text { Sin diagnóstico de VIH } \\
\qquad(n=563)\end{array}$} & \multicolumn{3}{|c|}{ Con diagnóstico de VIH $(n=627)$} \\
\hline & Media & DE & Media & DE & $t$ \\
\hline Temas sexuales & 4,46 & 0,76 & 4,26 & 0,86 & $2,72^{\star}$ \\
\hline Comunicación sexual con la pareja & 4,35 & 0,88 & 4,21 & 0,98 & $2,52^{*}$ \\
\hline
\end{tabular}

Nota. $* p<0,01$. Fuente: elaboración propia.

\section{Discusión}

El objetivo de la presente investigación consistió en validar dos escalas de comunicación sexual con la pareja basada en la propuesta de Dixson (1995). Para lograr el objetivo, se llevaron a cabo dos fases, en la primera de ellas se aplicó la estrategia de RSNM (Reyes-Lagunes, 1993), que considera que no basta con hacer un análisis de la literatura para el diseño de reactivos de un instrumento de medición, sino que, además, se debe contemplar a la población de interés de tal forma que tenga mayor certeza de contar con un instrumento culturalmente válido, para lo cual se debe solicitar a un grupo de personas que, a partir de un concepto central, elaboren una lista de palabras para definirlo. Con base en ello se puede conocer el significado que le atribuyen, así como las conductas que las personas consideran pertenecen al constructo de 
interés. Dicha estrategia ha mostrado evidencia empírica sobre la construcción de instrumentos (Bouquet et al., 2019; Campos-Rivera y Sotelo-Quiñonez, 2019; CapillaGarrido et al., 2020; Enríquez et al., 2021; Fonseca et al., 2019; García-Torres et al., 2017; Granados, 2019; Irepan y Ortega, 2020; Reyes et al., 2017; Rosales et al., 2019).

Con esta técnica se utilizaron dos estímulos para preguntar a los hombres con y sin diagnóstico de VIH que definieran temas sexuales y comunicación sexual con la pareja; de igual forma se cumplió con el criterio de que en cada grupo haya al menos 50 personas, y en el caso de la presente investigación fueron 100 hombres por grupo que permita cumplir con un rigor metodológico más estricto en la representación del constructo de interés (Hinojosa, 2008).

Los resultados obtenidos muestran que, en función del grupo al que pertenecen los hombres, aunque se observan diferencias cualitativas respecto a los temas que mencionan, al hacer la clasificación en términos de su carga afectiva, no hay diferencias entre los grupos. Un aspecto importante es que para las personas con VIH, en primer lugar, hablar de ITS se vincula con el contexto en el que se ubican y en el que se considera de mayor vulnerabilidad por el estigma y discriminación (Guevara-Sotelo y Hoyos-Hernández, 2018), en segundo lugar, está el sexo, que finalmente es relevante debido a que todos tienen una vida sexual activa, aunque cabe señalar que el condón ocupó el lugar seis en su lista. Esto es relevante, ya que en personas con VIH el uso del condón es primordial para evitar nuevas infecciones (Francis et al., 2018; Francis et al., 2019).

En el caso de las personas sin VIH hablar de sexo y condón estuvieron dentro de los primeros tres lugares, y amor ocupó el segundo lugar. Se observa que estos participantes mencionan palabras referentes a aspectos centrados en la relación afectiva y sexual en la relación de pareja, mientras que los hombres con VIH están orientados en aspectos de prevención, pero en ambos casos con una valoración positiva. Estos atributos son relevantes y deben ser tomados en cuenta para que los hombres puedan hablar con la pareja sexual independientemente de su estado serológico (Fonner et al., 2020).

Sobre el estímulo de comunicación sexual con la pareja, los hombres con VIH mencionan confianza, protección y responsabilidad. Nuevamente se observa que son aspectos relacionados con la protección sexual; mientras que los hombres sin diagnóstico de VIH mencionan transparencia, confianza, preguntas, apertura, entre otros, que describen más lo que se espera de la comunicación asertiva en pareja (Darden et al., 2019), aunque también señalan métodos anticonceptivos, VIH y sobre tener sexo; de tal forma que se centran en aspectos positivos de la comunicación con la pareja. De acuerdo con Reyes-Lagunes (1993) el que ambos grupos consideren tanto los 
temas, como la comunicación como un aspecto positivo y al no haber diferencias entre los grupos, respecto a ello, se decidió construir una escala en la que se contemplaran las palabras otorgadas por cada grupo.

Con base en lo anterior, se diseñaron reactivos para construir dos escalas de medición, la primera de ellas para evaluar los temas sexuales, y la segunda centrada en la comunicación sexual con la pareja. Para ello, se realizó un AFE y un AFC, ya que, de acuerdo con Schumacker y Lomax (2016) y Lloret-Segura et al. (2014), es necesario tomar en consideración ambos procesos para tener mayor certeza de la validez de constructo y los resultados obtenidos muestran evidencia de ello.

Particularmente, sobre los factores obtenidos, en el caso de la escala de temas sexuales, se divide en dos, en el primero de ellos son temas centrados en la salud sexual y el segundo sobre tener relaciones sexuales. Esto es relevante debido a que la literatura reporta la importancia para la prevención de infecciones por VIH, el que las personas hablen sobre tener relaciones sexuales y sobre cómo protegerse en esos encuentros (Gause et al., 2018), de tal forma que contar con un instrumento que permita identificar estos dos componentes dará indicadores sobre qué aspectos se deberán considerar en programas de intervención orientados a promover la salud sexual de estos hombres. Un aspecto característico de la muestra evaluada es que en su mayoría son hombres que se asumen como homosexuales, de tal forma que la orientación sexual es un tema relevante para ellos; valdría la pena analizar en fututos estudios qué sucede en población heterosexual, ya que diversos autores mencionan que la orientación sexual se relaciona con el cuidado de la salud sexual, estando más vulnerable la población homosexual o bisexual (Brooks, et al., 2018).

Por su parte, la escala de comunicación sexual con la pareja obtuvo dos factores, el primero de ellos sobre el estilo de comunicación asertiva. Si bien, los estilos de comunicación pueden ser asertivo, pasivo y agresivo (Longmire-Avital, 2019), se consideró en la presente investigación únicamente el estilo asertivo, ya que este es considerado como un factor protector que coadyuva en el cuidado de la salud sexual (Struckman-Johnson, Anderson y Smeaton, 2020).

El segundo factor, se relaciona con la percepción que tienen los hombres sobre el contexto en el que se da la comunicación sexual, que considera la importancia de hacerlo y cómo se siente al hablar con la pareja. De acuerdo con Aholou et al. (2017) esto es primordial y se refleja en el estudio en que evaluaron, en una muestra de hombres que tienen sexo con hombres, las percepciones sobre las conversaciones que tienen con su pareja respecto a la prevención del VIH y encontraron que las barreras percibidas para hablar del tema, dejar pasar el tema y no hablarlo, así como las emociones negativas, tienen un impacto en la salud sexual y en la forma de protegerse 
en las relaciones sexuales, por lo que preguntar al respecto podrá brindar indicadores que permitan que haya un contexto más favorable para que se dé la comunicación sexual con la pareja.

Como se puede observar, la validación de estas dos escalas puede brindar elementos para entender el proceso de comunicación sexual con la pareja; como ya se mencionó, se presentan evidencias de validez de constructo y de confiabilidad interna. De acuerdo con Martínez, Hernández y Hernández (2006), es necesario presentar la mayor evidencia de fuentes de validez, es por ello que se presentan los resultados de la validez concurrente, que señala la posible relación positiva o negativa entre constructos, en donde existe evidencia empírica. Por un lado, la escala de temas sexuales y de comunicación sexual con la pareja, de acuerdo con Dixson (1995), menciona que hay una relación positiva entre los componentes de contenido, estilo y contexto, y se puede constatar en la investigación de Cox et al. (2008); por otro lado, también se ha encontrado que hablar sobre temas sexuales, la comunicación asertiva y la percepción que se tiene de ella se relaciona con conductas sexuales seguras, como es el uso del condón (Aholou et al., 2017; Struckman-Johnson et al., 2020).

Un punto importante a considerar es que a pesar de encontrar una relación significativa que muestra evidencia de validez concurrente, las correlaciones entre la consistencia del uso del condón y los factores de las escalas es baja, por lo cual se debe prestar atención a si la manera en que se evaluó el uso del condón es la más pertinente, ya que de acuerdo con Gause et al. (2018) la medida que generalmente se utiliza más es la frecuencia del uso del condón, aspecto que sería conveniente retomar en futuras investigaciones para probar esta relación, que se observa en diversas investigaciones (Afriyanti et al., 2020; Crosby et al., 2016; Lo et al., 2011).

Con respecto al proceso de confiabilidad, en ambas escalas se obtuvieron propiedades psicométricas adecuadas, además se enriqueció el análisis con dos indicadores: el coeficiente alfa de Cronbach y el coeficiente omega de McDonald, ya que a pesar de que el primero es de los más utilizados, en los últimos años ha habido controversia respecto a su análisis, de tal manera que se propone el segundo como una forma de solventar estos errores (Martínez et al., 2006; Ventura-León y CaychoRodríguez, 2017). En ambos casos, se obtuvieron valores aceptables de confiabilidad, ya que, de acuerdo con Campo-Arias y Oviedo (2008), el índice debe encontrarse entre 0,70 y 0,90. y, en el caso particular de las dos escalas, los coeficientes estuvieron entre 0,84 y 0,91 .

Continuando con los resultados, también se presenta un análisis de diferencias entre el grupo con y sin diagnóstico de VIH, si bien se encontró que es mayor la frecuencia con la que hablan de temas sexuales y es más la frecuencia del estilo 
asertivo, así como una percepción más favorable del contexto en los hombres sin VIH, se debe poner atención sobre el hecho de que a pesar de que esta diferencia es estadísticamente significativa, al observar cualitativamente el valor obtenido en las medias, la diferencia no es tan grande, una explicación sobre esto es que en la presente investigación, la aplicación de los instrumentos se realizó en una clínica especializada de atención de VIH, de tal forma que si bien, hay hombres que acuden a recibir su tratamiento de $\mathrm{VIH}$, otros acuden a realizarse una prueba de detección de $\mathrm{VIH}$, lo que hace suponer que comparten ciertas características e inclusive algunos de ellos saldrán con su diagnóstico de VIH. En este sentido se recomienda aplicar estas escalas en hombres que nunca se han hecho una prueba de detección, así como en hombres sin diagnóstico de VIH en los que haya pasado tiempo desde su última prueba, ya que es probable que esto pudiera influir en las respuestas otorgadas. En este mismo sentido, se recomienda a futuras investigaciones evaluar otras muestras de hombres, si bien el criterio de inclusión fue hombres que reportaran tener sexo con hombres, en su mayoría tuvieron una orientación sexual homosexual, por lo que también sería interesante poder hacer comparaciones entre su orientación sexual, e inclusive con hombres que no tienen sexo con hombres.

Por último, de acuerdo con la propuesta de Dixson (1995) en la presente investigación se consideró de manera explícita tres de los cinco componentes que proponen: el contenido, el estilo y el contexto. Respecto a los otros dos componentes; sobre la extensión, que se refiere a la frecuencia con la que se da la comunicación, implícitamente se consideró en las opciones de respuesta, que permite evaluar la frecuencia con la que se comunican con la pareja; y el tiempo, es decir, sobre el momento en el que da la comunicación, para el que se tomó en cuenta el diagnóstico de VIH, es decir, cómo es la comunicación con la pareja en los hombres antes y después del diagnóstico, de tal forma que los instrumentos validados permiten valorar los cinco componentes de la comunicación.

En conclusión, se cumplió con el objetivo de la investigación, hasta el momento se cuenta con dos instrumentos válidos y confiables que permiten evaluar los componentes de la comunicación sexual con la pareja en hombres que tienen sexo con hombres y con diagnóstico de VIH; sin embargo, es necesario seguir corroborando las propiedades psicométricas del instrumento en muestras similares, y hacer evaluaciones considerando las comparaciones antes mencionadas, de tal forma que se contribuya en la explicación de cómo la comunicación coadyuva en el uso del condón en relaciones sexuales y, con base en ello, diseñar intervenciones para promover habilidades centradas en mejorar la comunicación sexual. 


\section{Ricardo Sánchez Medina, David Javier Enríquez Negrete, Consuelo Rubi Rosales Piña, 23}

\section{Referencias}

Abad, F., Olea, J., Ponsoda, V. y García, C. (2011). Medición en ciencias sociales y de la salud. Síntesis.

Afriyanti, L., Waluyo, A. \& Yona, S. (2020). Correlations between Drug Use, HIV Disclosure and Interpersonal Communication on Sexual Risk Behaviour of HIv-Positive Men Who Have Sex with Men. BMC Proceedings, 14(13), 20. https://doi.org/10.1186/s12919-020-00200-5

Aholou, T., Nanin, J., Drumhiller, K. \& Sutton, M. (2017). Opportunities for HIV Prevention Communication during Sexual Encounters with Black Men Who have Sex with Men. AIDS Patient Care and STDs, 31(1), 33-40. http://doi.org/10.1089/apc.2016.0220

Ali, M. S., Tesfaye Tegegne, E., Kassa Tesemma, M. y Tesfaye Tegegne, K. (2019). Consistent Condom Use and Associated Factors Among HIV-Positive Clients on Antiretroviral Therapy in North West Ethiopian Health Center, 2016 GC. AIDS Research and Treatment, 2019, 1-10. https://doi. org/10.1155/2019/7134908

Altice, F., Evuarherhe, O., Shina, S., Carter, G. \& Beaubrun, A. C. (2019). Adherence to HIV treatment regimens: systematic literature review and meta-analysis. Patient Preference \& Adherence, 13, 475-490. https://doi.org/10.2147/PPA.S192735

Álvarez, C., \& Villaruel, A. (2015). Association of Gender Norms, Relationship and Intrapersonal Variables, and Acculturation with Sexual Communication among young Adult Latinos. Research in Nursing \& Health, 38(2), 121-132. https://doi.org/10.1002/nur.21645

Anderson, A., Rosen, N., Price, L., \& Bergeron, S. (2016). Associations Between Penetration Cognitions, Genital Pain, and Sexual Well-Being in Women with Provoked Vestibulodynia. The Journal of Sexual Medicine, 13, 444-452. https://doi.org/10.1016/j.jsxm.2015.12.024

Araujo-Robles, E., Ucedo-Silva, V. y Bueno-Cuadra, R. (2018). Validación de la Escala de Comunicación Padres-Adolescentes en jóvenes universitarios de Lima. Revista Digital de Investigación y Docencia Universitaria, 12(1), 253-272. https://doi.org/10.19083/ridu.12.560

Ayer, A., Perez-Brumer, A., Segura, E. R., Chavez-Gomez, S., Fernandez, R., Arroyo, C., Barrantes, A., Lake, J. E., Cabello, R. \& Clark, J. L. (2021). Let's Talk About Sex: The Impact of Partnership Contexts on Communication About HIV Serostatus and Condom Use Among Men Who Have Sex with Men (MSM) and Transgender Women (TW) in Lima, Peru. AIDs and Behavior, 25(7), 21392153. https://doi.org/10.1007/s10461-020-03144-1 
Diseño y validación de dos escalas de comunicación sexual con la pareja en hombres que tienen sexo con hombres

Blunt-Vinti, H., Jozkowki, K. \& Hunt, M. (2019). Show or Tell? Does Verbal and/or Nonverbal Sexual Communication Matter for Sexual Satisfaction? Journal of Sex \& Marital Therapy, 45(3), 206217. https://doi.org/10.1080/0092623X.2018.1501446

Bouquet, G. S., García, M., Díaz, R. y Rivera, S. (2019). Conceptuación y Medición de la Agresividad: Validación de una Escala. Revista Colombiana de Psicología, 28(1), 115-130. https://doi. org/10.15446/rcp.v28n1.70184

Brooks, H., Llewellyn, C. D., Nadarzynski, T., Pelloso, F. C., De Souza Guilherme, F., Pollard, A. \& Jones, C. J. (2018). Sexual Orientation Disclosure in Health Care: A Systematic Review. British Journal of General Practice, 68(668), 187-196. https://doi.org/10.3399/bjgp18X694841

Campo-Arias, A. y Oviedo, H. C. (2008). Propiedades psicométricas de una Escala: la Consistencia Interna. Revista de Salud Pública, 10(5), 831-839.

Campos-Rivera, N. y Sotelo-Quiñonez, T. (2019). Diseño y validación de una escala de actitudes maternas hacia el sobrepeso y la obesidad infantil. Acta Colombiana de Psicología, 22(2), 148-162. http://dx.doi.org/10.14718/ACP.2019.22.2.8

Capilla-Garrido, E., Cubo-Delgado, S. y Gutiérrez-Esteban, P. (2020). Diseño y validación de un instrumento para evaluar los comportamientos phubbing. RELIEVE, 26(2), 1-16. http://doi. org/10.7203/relieve.26.2.16955

Carrascosa, L., Cava, M. y Buelga, S. (2015). Actitudes hacia la autoridad, y violencia entre adolescentes: diferencias en función del sexo. Suma Psicología, 22, 102-109. https://doi.org/10.1016/j.sumpsi.2015.08.003

Cox, M. F., Fasolino, T. K. \& Tavakoli, A. S. (2008). Factor Analysis and Psychometric Properties of the Mother-Adolescent Sexual Communication (MASC) Instrument for Sexual Risk Behavior. Journal of Nursing Measurement, 16(3), 171-183. https://doi.org/10.1891/1061-3749.16.3.171

Crosby, R. A., Graham, C. A., Yarber, W. L., Sanders, S. A., Milhausen, R. R. \& Mena, L. (2016). Measures of Attitudes Toward and Communication about Condom Use: Their Relationships with Sexual Risk Behavior among Young Black Men Who have Sex with Men. Sexually Transmitted Diseases, 43(2), 94-98. https://doi.org/10.1097/OLQ.00000000000000392

Damian, D. J., Ngahatilwa, D., Fadhili, H., Mkiza, J. G., Mahande, M. J., Ngocho, J. S., \& Msuya, S. E. (2019). Factors Associated with HIV Status Disclosure to Partners and its Outcomes Among HIV-Positive Women Attending Care and Treatment Clinics at Kilimanjaro region, Tanzania. PLoS One, 14(3), e0211921. https://doi.org/10.1371/journal.pone.0211921 
Darden, M. C., Ehman, A. C., Lair, E. C. \& Gross, A. M. (2019). Sexual Compliance: Examining the Relationships among Sexual Want, Sexual Consent, and Sexual Assertiveness. Sexuality \& Culture, 23, 220-235. https://doi.org/10.1007/s12119-018-9551-1

Dharma, C., Scheim, A. I. \& Bauer, G.R. (2019). Exploratory Factor Analysis of Two Sexual Health Scales for Transgender People: Trans-Specific Condom/Barrier Negotiation Self-Efficacy (T-Barrier) and Trans-Specific Sexual Body Image Worries (T-Worries). Archives of Sexual Behavior, 48, 1563-1572. https://doi.org/10.1007/s10508-018-1383-4

DiClemente, R. \& Wingood, G. (1995). A Randomized Controlled Trial of an HIv Sexual Risk Reduction Intervention for Young African-American Women. Journal of the American Medical Association, 274(16), 1271-1276.

Dimbuene, Z. (2015). Families' Response to AIDS: New Insights into Parental Role in Fostering HIV/AIDS Knowledge. Journal of Biosocial Science, 47, 762-779. https://doi.org/10.1017/ S0021932014000406

Dixson, M. D. (1995). Models and Perspectives of Parent-Child Communication. In T. J. Socha \& G. H. Stamp (Eds.), Parents, Children, \& Communication: Frontiers of Theory and Research (pp. 43-61). Lawrence Erlbaum Associates.

Enríquez, D., Sánchez, R., Bárcena, S. X., Echeverría, I. S. y Arias, B. D. (2021). Construcción y validación de una escala de socialización sexual paterna por dominios. Psicología UNEMI, 5(9), 71-84. https://doi.org/10.29076/issn.2602-8379vol5iss9.2021pp71-84p

Esparza, S., Dimmitt, J., Moreno, M., Tovar, M. y Flores, M. (2017). Mexicans Adolescents' Self-Reports of Parental Monitoring and Sexual Communication for Prevention of Sexual Risk. Journal of Pediatric Nursing, 35, 83-89. https://doi.org/10.1016/j.pedn.2017.03.007

Fernández, A., McFarlen, M., González, R., Díaz, L., Betancourt- Díaz, E., Cintrón- Bou, F., Varas-Díaz, N. y Villaruel, A. (2017). Actitudes hacia la comunicación sexual entre padres/madres y adolescentes en Puerto Rico. Revista Puertorriqueña de Psicología, 28(1), 80-95.

Fonner, V., Mbwambo, J., Kennedy, C., Kerrigan, D. \& Sweat, M. (2020). Do Sexual Partners Talk to each other about HIV? Exploring Factors Associated with HIV-Related Partner Communication among Men and Women in Tanzania. AlDS Behavior, 24, 891-902. https://doi.org/10.1007/ s10461-019-02550-4

Fonseca, J., Cruz, C. y Chacón, L. (2019). Validación del instrumento de compromiso organizacional en México: evidencias de validez de constructo, criterio y confiabilidad. Revista de Psicología, 37(1), 7-29. https://dx.doi.org/10.18800/psico.201901.001 
Diseño y validación de dos escalas de comunicación sexual con la pareja en hombres que tienen sexo con hombres

Francis, D. B., Zelaya, C. M., Fortune, D. A. \& Noar, S. M. (2019). Black College Women's Interpersonal Communication in Response to a Sexual Health Intervention: A Mixed Methods Study. Health communication, 1-9. https://doi.org/10.1080/10410236.2019.1673949

Francis, D., Noar, S., Fortune, D. \& Adimora, A. (2018). "Be Straight Up and So Will He": Evaluation of a Novel HIv Prevention Condom Distribution and Health Communication Intervention Targeting Young African American Females. AIDS Education and Prevention, 30(2), 137-151. https://doi.org/10.1521/aeap.2018.30.2.137

Gabbidon, K., Shaw-Ridley, M. \& George, F. (2017). Relationship between Culture and Parent-Teen Sexuality Conversations in Black Families. Californian Journal of Health Promotion, 15(2), 1326. https://doi.org/10.32398/cjhp.v15i2.1896

García-Torres, M., García-Méndez, M. y Rivera-Aragón, S. (2017). Apoyo social en adultos mexicanos: validación de una escala. Acta de investigación psicológica, 7(1), 2561-2567. https://doi. org/10.1016/j.aipprr.2017.02.004

Gause, N. K., Brown, J. L., Welge, J. \& Northern, N. (2018). Meta-Analyses of HIV Prevention Interventions Targeting Improved Partner Communication: Effects on PartnerCommunication and Condom Use Frequency Outcomes. Journal of Behavioral Medicine, 41, 423-440. https:// doi.org/10.1007/s10865-018-9916-9

González,V., Orcasita, L., Carrillo, J. y Palma-García, D. (2017). Comunicación familiar y toma de decisiones entre ascendientes y adolescentes. Revista Latinoamericana de Ciencias Sociales, 15(1), 419-430.

Granados, R. (2019). Validación psicométrica de la escala de valoración de riesgo de violencia en adolescentes. Archivos de Criminología, Seguridad Privada y Criminalística, 22, 107-12.

Grimm, J. \& Schwartz, J. (2021). PrEP, HIV, and the importance of health communication. In S. Bernays, A. Bourne, S. Kippax, P. Aggleton, \& R. Parker (Eds.), Remaking HIV Prevention in the 21st Century: The Promise of TasP, U=U and PrEP (pp. 47-58). Springer International Publishing. https://doi.org/10.1007/978-3-030-69819-5_4

Guevara-Sotelo, Y. y Hoyos-Hernández, P. A. (2018). Vivir con VIH: experiencias de estigma sentido en personas con VIH. Psicogente, 21(39), 127-139. http://doi.org/10.17081/psico.21.39.2827

Gursahaney, P. R., Cordes, S., Ofotokun, I., Wall, K. M., Jamieson, D. J. \& Haddad, L. B. (2019). Factors Associated with Condom Use among HIV-Positive Women Living in Atlanta, Georgia. PLoS One, 14(12), e0225406. https://doi.org/10.1371/journal.pone.0225406 
Haas, S. M., Perazzo, J. D., Ruffino, A. H., Ancona, R. M. \& Lyons, M. (2017). The KnowNow Project: Facilitated Serosorting in HIV-Status Sexual Partner Communication. AIDS Education and Prevention, 29(5), 432-442. https://doi.org/10.1521/aeap.2017.29.5.432

Haas, S. M., Perazzo, J. D., Ruffner, A. H. \& Lyons, M. S. (2020). Exploring Current Stereotypes and Norms Impacting Sexual Partner HIv-Status Communication. Health Communication, 35(11), 1376-1385. https://doi.org/10.1080/10410236.2019.1636340

Harris, A. (2016). African American Parent-Son Sexual Communication among a College Sample. Journal of Pediatric Nursing, 31, 199-206. https://doi.org/10.1016/j.pedn.2015.12.006

Hinojosa, G. (2008). El tratamiento estadístico de las redes semánticas naturales. Revista Internacional de Ciencias Sociales y Humanidades, XVIII(1),133-154.

Iglesias-García, M., Urbano-Contreras, A. y Martínez-González, R. (2019). Escala de Comunicación Autopercibida en la Relación de Pareja (CARP). Anales de Psicología, 35(2), 314-322. https:// doi.org/10.6018/analesps.35.2.334451

Irepan, M. y Ortega, P. (2020). Desarrollo y validación de la escala de restauración psicológica percibida en población mexicana. Acta de investigación psicológica, 10(1), 80-90. https://doi. org/10.22201/fpsi.20074719e.2020.1.335

Jones, A., Robinson, W. \& Seedall, R. (2017). The Role of Sexual Communication in Couples' Sexual Outcomes: A Dyadic Path Analysis. Journal of Marital and Family Therapy, 44(4), 606-623. https://doi.org/10.1111/jmft.12282

Kerlinger, F. y Lee, H. (2002). Investigación del comportamiento. Métodos de investigación en ciencias sociales. McGraw Hill.

Leonhardt, N., Willoughby, B., Busby, D., Yorgason, J., \& Holmes, E. (2018). The Significance of The Female Orgasm: ANationally Representative, Dyadic Study of Newlyweds' Orgasm Experience. The Journal of Sexual Medicine, 15(8), 1140-1148. https://doi.org/10.1016/j.jsxm.2018.05.018

Li,Y.\&Samp, J.A. (2019). Sexual Relationship Power, Safer Sexual Communication, and Condom Use: A Comparison of Heterosexual Young Men and Women. Western Journal of Communication, 83(1), 58-74. https://doi.org/10.1080/10570314.2017.1398835

Lloret-Segura, S., Ferreres-Traver, A., Hernández-Baeza, A., y Tomás-Marco, I. (2014). El análisis factorial exploratorio de los ítems: una guía práctica, revisada y actualizada. Anales de Psicología, 30(3), 1151-1169. 
Diseño y validación de dos escalas de comunicación sexual con la pareja en hombres que tienen sexo con hombres

Lo, S. C., Reisen, C. A., Poppen, P. J., Bianchi, F. T. \& Zea, M. C. (2011). Cultural Beliefs, Partner Characteristics, Communication, and Sexual Risk among Latino MSM. AIDS and Behavior, 15(3), 613-620. https://doi.org/10.1007/s10461-010-9760-6

Longmire-Avital, B. (2019). "I Asked for the Papers": How Emerging Adult Black Women Request Sexual Health Information. Journal of Black Sexuality and Relationships, 6(1), 29-48.

López-Olmos, J. (2018). Hijas y madres. Resultados en la comunicación de temas sexuales y reproductivos. Revista de Medicina e Investigación, 6(2), 18-34.

López, J. (2019). Epidemiología y control de las infecciones de transmisión sexual. Papel de las unidades de ITS. Enfermedades Infecciosas y Microbiología Clínica, 37(1), 45-49. https://doi. org/10.1016/j.eimc.2018.10.015

Martínez-Huertas, J. A. y Jastrzebska, O. (2019). Adaptación Española de la Escala de Comunicación Diádica Sexual. Psicología Conductual, 27(2), 311-324.

Martínez, M., Hernández, M. y Hernández, M. (2006). Psicometría. Alianza Editorial.

Megaputri, P., Prima, P., Sari, C. \& Rismayanti, I. (2019). Deceptive is Effective Negotiation Strategies to Customers For Using Condoms Consistently. INSIST, 2(2), 93-96. http://dx.doi.org/10.23960/ ins.v2i2.88

Mengual, V. J. M., Llario, M. D. G., Arnal, R. B. y Rodríguez, I. M. D. (2016). Conductas de riesgo y actitudes relacionadas con la infección por VIH/SIDA en HSH. International Journal of Developmental and Educational Psychology. Revista INFAD de Psicología, 3(1), 409-414.

Montero, I. \& León, O. (2007). A Guide for Naming Research Studies in Psychology. International Journal of Clinical and Health Psychology, 7(3), 847-862.

Muhwezi, W., Katahoire, A., Banura, C., Mugooha, H., Kwesiga, D., Bastien, S. \& Kleep, K. (2015). Perceptions and Experiences of Adolescents, Parents and School Administrators Regarding Adolescents-Parents Communication on Sexual and Reproductive Health Issues in Urban and Rural Uganda. Reproductive Health, 12, 110. https://doi.org/10.1186/s12978-015-0099-3

Ncube, B., Ansong, J., Daniels, K., Campbell-Stennett, D. \& Jolly, P. (2017). Sexual Risk Behavior Among HIv-Positive Persons in Jamaica. African Health Sciences, 17(1), 32-38. https://doi. org/10.4314/ahs.v17i1.6

Olaz, F. (2014). Confiabilidad. En S. Tornimbeni, E. Pérez y F. Olaz (Eds.), Introducción a la psicometría (pp. 71-100). Paidós. 
Organización Mundial de la Salud. (oms). (2019). Infecciones de Transmisión Sexual. https://bit. ly/3lsOUEy

Otero-Guevara, L., Fernández-Blázquez, A. y Vázquez, F. (2017). Diagnóstico rápido de las infecciones de transmisión sexual. Enfermedades Infecciosas y Microbiología Clínica, 35(7), 444-450. https://doi.org/10.1016/j.eimc.2017.01.004

Parker, S. M., Clayton, J. M., Hancock, K., Walder, S., Butow, P. N., Carrick, S., Currow, D., Ghersi, D., Glare, P., Hagerty, R. \& Tattersall, M. H. N. (2007). A systematic review of prognostic/end-of-life communication with adults in the advanced stages of a life-limiting illness: Patient/caregiver preferences for the content, style, and timing of information. Journal of Pain and Symptom Management, 34(1), 81-93. https://doi.org/10.1016/j.jpainsymman.2006.09.035

Pazmany, E., Bergeron, S., Verhaeghe, J., Van Oudenhove, L. y Enzlin, P. (2015). Dyadic Sexual Communication in Pre-Menopausal Women with Self-Reported Dyspareunia and their Partners: Associations with Sexual Function, Sexual Distress and Dyadic Adjustment. The Journal of Sexual Medicine, 12(2), 516-528. https://doi.org/10.1111/jsm.12787

Peasant, C., Montanaro, E., Kershaw, T. Parra, G., Weiss, N., Meyer, J., Murphy, J., Ritchwood, T. \& Sullivan, T. (2017). An Event-Level Examination of Successful Condom Negotiation Strategies among Young Women. Journal of Health Psychology, 24(7), 898-908. https://doi. org/10.1177/1359105317690598

Pérez, O. (2015). Redes semánticas naturales: anotaciones metodológicas para el análisis de las representaciones sociales. Revista Contribuciones a las Ciencias Sociales, 27.

Programa Conjunto de las Naciones Unidas sobre el VIH/SIDA. (ONUSIDA). (2020). Hoja informativa - Últimas estadísticas sobre el estado de la epidemia de sida. https://bit.ly/3oTg9KH

Quinn-Nilas, C., Milhausen, R., Breuer, R., Bailey, J., Pavlou, M., DiClemente, R. \& Wingood, G. (2015). Validation of the Sexual Communication Self-Efficacy Scale. Health Education \& Behavior, 43(2), 165-171. https://doi.org/10.1177/1090198115598986

Rancourt, K., Flynn, M., Bergeron, S. \& Rosen, N. (2017). It Takes Two: Sexual Communication Patterns and the Sexual and Relational Adjustment of Couples Coping with Provoked Vestibulodynia. The Journal of Sexual Medicine, 14(3), 434-443. https://doi.org/10.1016/j.jsxm.2017.01.009

Rancourt, K., Rosen, N., Bergeron, S., \& Nealis, L. (2016). Talking about sex when sex is painful: dyadic sexual communication is associated with women's pain, and couples' sexual and psychological outcomes in provoked Vestibulodynia. Archives of Sexual Behavior, 45(8), 1933-1944. https://doi.org/10.1007/s10508-015-0670-6 
Diseño y validación de dos escalas de comunicación sexual con la pareja en hombres que tienen sexo con hombres

Reidl-Martínez, L. (2013). Confiabilidad en la medición. Investigación en Educación Médica, 2(6), 107-111. https://doi.org/10.1016/s2007-5057(13)72695-4

Reyes-Lagunes, I. (1993). Redes semánticas para la construcción de instrumentos. Revista de Psicología Social y Personalidad, 9(1), 83-99.

Reyes, J.D., Rivera, L., Tellez, M. A., González, E., Galindo, Ó. y Seyler, A. (2017). Construcción y validación de un instrumento de violencia en el noviazgo en parejas heterosexuales y homosexuales. Revista Electrónica de Psicología Iztacala, 20(2), 581-605.

Rogers, A. (2017). Parent-Adolescent Sexual Communication and Adolescents' Sexual Behaviors: A Conceptual Model and Systematic Review. Adolescent Research Review, 2, 293-313. https:// doi.org/10.1007/s40894-016-0049-5

Rogers, A., Padilla-Walker, L. \& Hurst, J. (2020). Development and Testing of Parent-Child Sex Communiaction Inventory: A Multidimensional Assessment Tool for Parent and Adolescent Informants. The Journal of Sex Research, 1-14. https://doi.org/10.1080/00224499.2020.179 2398

Rosales, C., Sánchez, R. y Muñoz, S. (2019). Escala de aprendizaje autogestivo en estudiantes universitarios de la carrera de psicología de un sistema en línea. Hamut’ay, 6(2), 102-125. http:// dx.doi.org/10.21503/hamu.v6i2.1778

Sánchez, R., Enríquez, D. y Rosales, R. (2018). Intervención para promover habilidades de negociación del uso del condón en hombres privados de la libertad. En M. Molero, M. Pérez-Fuentes, J. Gázquez, M. Simón, A. Barragán, A. Martos y M. Sisto. Intervención en Contextos Clínicos y de la Salud (Vol. II, pp. 291-296). ASUNIVEP.

Santos-Iglesias, P. \& Byers, E. S. (2020). Development and initial validation of the verbal and nonverbal sexual communication questionnaire in Canada and Spain. Sexual and Relationship Therapy, 35(1), 60-76. https://doi.org/10.1080/14681994.2018.1442569

Schumacker, R. \& Lomax, R. (2016). Beginner's Guide to Structural Equation Modeling. Routledge.

Soto, V. (2015). Infecciones de transmisión sexual: epidemiología y prevención. Revista Experiencia en Medicina, 1(2), 61-65.

Struckman-Johnson, C., Anderson, P. B. \& Smeaton, G. (2020). Predictors of female sexual aggression among a U.S. MTurk Sample: The protective role of sexual assertiveness. Journal of Contemporary Criminal Justice. https://doi.org/10.1177/1043986220936100 
Tuason, I. C., Bernarte, R. \& Dong, F. (2017). The Relationship of Religion, Religiosity, and Parental Communication in The Sexual Behaviors of Filipinos aged 18-25 years in the United States and the Philippines. Asian/Pacific Island Nursing Journal, 2(4), 124-132. https://doi. org/10.9741/23736658.1060

Uribe, A., Castellanos, J. y Cabán, M. (2016). Conductas sexuales de riesgo y comunicación sobre sexualidad entre padres e hijos universitarios. Revista de Psicología Universidad de Antoquía, $8(2), 27-48$.

Valdez, M. J. L. (1998). Las redes semánticas naturales. Usos y aplicaciones en Psicología Social. Universidad Autónoma del Estado de México.

Ventura-León, J. y Caycho-Rodríguez, T. (2017). El coeficiente Omega: un método alternativo para la estimación de la confiabilidad. Revista Latinoamericana de Ciencias Sociales, Niñez y Juventud, 15(1), 625-627.

Vidal, E. y Hernández, B. (2017). Conductas sexuales de riesgos a las infecciones de transmisión sexual en adolescentes de una comunidad. Revista Habanera de Ciencias Médicas, 16(4), 625-634.

Widyatuti, Hafilah, C. \& Yuni, A. (2018). Correlation between Parent-Adolescent Communication and Adolescents' Premarital Sex Risk. Enfermería clínica, 28(1)51-54. https://doi.org/10.1016/ S1130-8621(18)30036-6 